\title{
From Multiple Organ Support Therapy to Extracorporeal Organ Support in Critically III Patients
}

\author{
Claudio Ronco a, b Zaccaria Riccic Faeq Husain-Syed ${ }^{d}$ \\ a Department of Nephrology, Dialysis and Transplantation, San Bortolo Hospital, Viale Ferdinando Rodolfi, Vicenza,

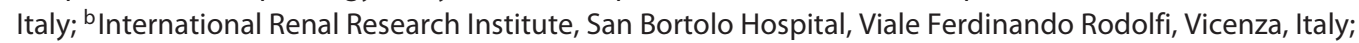 \\ 'Department of Cardiology and Cardiac Surgery, Pediatric Cardiac Intensive Care Unit, Bambino Gesù Children's

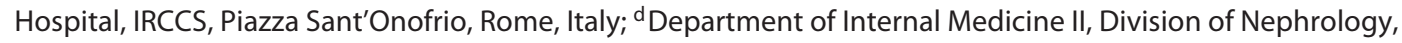 \\ Pulmonology and Critical Care Medicine, University Hospital Giessen and Marburg, Giessen, Germany
}

\section{Keywords}

Extracorporeal organ support - Extracorporeal membrane oxygenation · Multiorgan dysfunction syndrome $\cdot$ Renal replacement therapy

\begin{abstract}
Critically ill patients developing severe forms multiple organ dysfunction syndrome (MODS) may not be adequately supported by pharmacologic management. In these complex cases, a single form of extracorporeal organ support (ECOS) may be required, but multiple organ support therapy (MOST) is currently seen as a feasible approach. Severe renal dysfunction is a typical syndrome requiring renal replacement therapy (RRT) in the context of MODS. After more than a decade of RRT application in various intensive care settings, ECOS are not anymore seen as extraordinary or particularly aggressive techniques in MODS patients. Nowadays, a significant increase in the use of extracorporeal membrane oxygenation and extracorporeal carbon dioxide removal is occurring. When renal and cardio-pulmonary ECOS are used together, a multidisciplinary approach is necessary to minimize negative interactions and unwanted adverse effects. In this editorial, we focus on the organ crosstalk between the
\end{abstract}

\section{KARGER}

(c) 2019 S. Karger AG, Basel

E-Mail karger@karger.com

www.karger.com/bpu native and artificial organs, including the advantages and disadvantages of organ support on multiorgan function. Much of current experience on MOST has been gained upon RRT connected to other organ support therapies. Overall, available literature has not definitely established the ideal timing of these interventions, and whether early implementation impacts organ recovery and optimizes resource utilization is still a matter of open debate: it is possible that future research will be devoted to identify patient groups that may benefit from short- and long-term multiple organ support. Video JournalClub "Cappuccino with Claudio Ronco" at https:// www.karger.com/Journal/ArticleNews/490694?sponsor=52

(c) 2019 S. Karger AG, Basel

\section{Introduction}

The management of critically ill patients in the intensive care unit (ICU) is progressively increasing in complexity [1]. Significant advances in care, comorbidity and advanced age of patients led to a greater severity of illness at admission [2]. Simultaneous dysfunction of various organs is frequent, leading to the so called Multiple Organ Dysfunction/Failure Syndrome (MODS/MOFS) [3]. 
Fig. 1. Schematic representation of different native organ interaction and cross-talk. The injury to one organ may result in a secondary damage/dysfunction of other organs whose compromised function activates a vicious circle and a worsening of MODS.

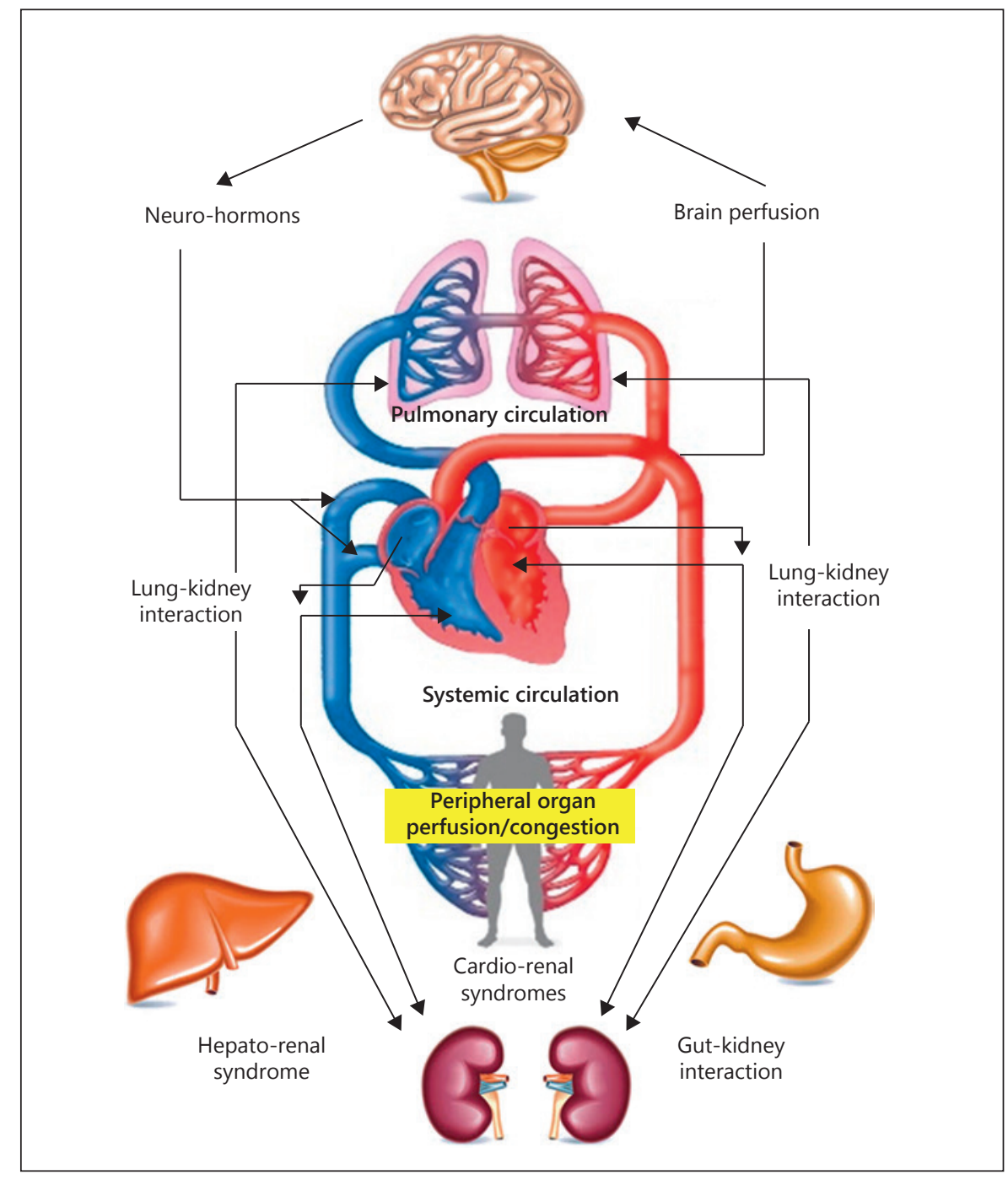

\section{The Complex Nature of MODS}

Several organ systems are involved in critical illness where the initial impairment of one organ function is often followed by dysfunction or damage in other organs. This is especially true in the context of sepsis or other systemic disorders [3]. For example, the effect of acute kidney injury (AKI) on distant organs is now well documented [4]. This phenomenon may be observed with a primary injury to other single organs followed by secondary damage/dysfunction of other organs (Fig. 1). The initial sequence of events often results in a vicious circle leading to a continuous negative organ interaction and a progressive worsening of the syndrome [5]. This is typically the case of cardio-renal syndromes where several bidirectional and temporally related heart-kidney interactions may lead to 5 different clinical subtypes [6]. The syn- drome initiation, the primary organ involved and the mechanisms are different in nature: haemodynamic alterations and congestion, iatrogenic effect of interventions, direct toxicity of drugs or contrast media, neurohormonal derangements and immune-mediated/inflammatory damage [7]. Nevertheless, after a significant organ cross-talk is initiated, the progressive dysfunction of both organs leads to significant worsening of the clinical picture.

Other conditions may involve acute and chronic lung disease scenarios leading to AKI or accelerated chronic kidney disease, and vice versa [8]. Some of these interactions include the participation of the heart in an even more complex cardio-pulmonary-kidney cross-talk [5].

Critically ill patients may develop liver dysfunction in the context of MODS or may be affected by primary liver disorders. Liver dysfunction may become the trigger for 
several pathological pathways, eventually involving lungs, kidneys and brain [9]. Combined liver and kidney dysfunction is common and described by different types of hepato-renal syndrome [10]. AKI represents a well-known complication of liver disease through different biological pathways and it is associated with increased morbidity and mortality. Renal dysfunction in cirrhosis is often functional in nature and secondary to haemodynamic derangements, cardiac dysfunction, and altered plasma composition. Nevertheless, an increasing number of patients with cirrhosis may develop structural damage of the kidneys leading to a progressive deterioration of organ function [11]. In turn, once kidney function deteriorates in liver patients, a progressive worsening of the syndrome is typically observed with unfavourable outcomes.

Gut and kidney may also present reciprocal negative interactions due to primary alterations in host microbiome profile and disruption of gut barrier function leading to systemic inflammation, AKI, progression of chronic kidney disease with effects on uremic toxicity and potential increase in cardiovascular risk [12]. On the other hand, the effects of AKI on the increased risk of bleeding and other derangements of the gastro-intestinal tract have been described [13].

All these syndromes are often the result of a mixture of direct organ injury, secondary systemic disorders and altered tissue perfusion in different organ systems. Pre-existing organ dysfunction can make the clinical picture worse [14]. Furthermore, primary and secondary organ injury/ dysfunction results from a complex balance between individual susceptibility and exposure (insult) intensity [15]. The interaction between these 2 factors is particularly evident in the case of sepsis where several organs are affected by an exaggerated and uncontrolled imbalance between the pro- and anti-inflammatory response of the host. The so-called immune-homeostasis is compromised and organ dysfunction is generally the result of altered blood perfusion and metabolism at the tissue and cellular level [16]. Although individual characteristics become less important when the intensity of exposure (insult) is overwhelming, the contribution of host response to organ injury may still be significant and precision medicine criteria should be applied for the final treatment strategy [3].

\section{Multiple Organ Support Therapy}

Critically ill patients with MODS require a complex and articulated therapeutic approach that includes pharmacological strategies (such as antibiotic for infection source control, circulatory and respiratory support, organ-specific drugs, correction of abnormalities of coagulation, electrolyte, acid-base, metabolism) and specific organ support systems. All these interventions should be integrated in a global strategy to support single organs and manage the combined effects of multiple organ crosstalk. In a seminal paper, we described the concept of multiple organ support therapy (MOST) identifying the possibility to provide simultaneous and combined support to different failing organ systems [17]. MOST includes oxygenation and ventilatory support (invasive and noninvasive mechanical ventilation $[\mathrm{MV}]$, veno-venous (VV) extracorporeal membrane oxygenation [ECMO] and extracorporeal carbon dioxide removal $\left[\mathrm{ECCO}_{2} \mathrm{R}\right]$ ), mechanical circulatory support (intra-aortic balloon pump, veno-arterial (VA) ECMO, percutaneous and surgical ventricular assist devices [VADs] and total artificial heart), renal replacement therapy (RRT) and extracorporeal liver support (molecular adsorbent recirculating system, plasmapheresis and sorbent therapies). Al these techniques are currently used in the ICU although very little is known about their interaction with native organs and other artificial organ support systems [18].

\section{Extracorporeal Organ Support}

Extracorporeal blood purification techniques such as haemodialysis or haemofiltration have been used successfully for several decades to replace renal function in critically ill patients with kidney failure. New applications are today emerging for extracorporeal techniques. The experience with extracorporeal blood therapies in sepsis, suggests that the spectrum of application be redefined and we are today exploring the concept of extracorporeal organ support (ECOS) to describe all forms of therapies where blood is extracted from the body and processed in different circuits with specific devices and techniques [19]. The principle for ECOS is that in MODS, failing organs have in common the blood perfusing their tissues, and circulating blood becomes the target for specific treatments.

The idea of using extracorporeal therapies for sepsis came from the occasional observation that septic patients treated with RRT for AKI displayed a rapid and significant improvement in haemodynamics, with a reduced requirement of vasopressor support few hours after the application of the extracorporeal circulation. Further experiments demonstrated that the ultrafiltrate recovered from septic patients treated with haemofiltration and injected 
in healthy animals produced septic symptoms [20]. The improvement in septic manifestations in patients undergoing RRT suggested a possible reduction of circulating chemical mediators eliminated in the ultrafiltrate. The absence of significant variation in circulating levels of cytokines created some conflicting positions [21] that will never be resolved until a well-designed and adequately powered trial on extracorporeal therapies in sepsis in the absence of AKI will be performed. Still, the question whether mortality is the correct endpoint for such study is wide open. Nevertheless, some hypotheses were formulated such as the peak concentration hypothesis [22] based on the idea that a non-selective elimination of the peaks of both pro- and anti-inflammatory mediators might contribute to a restoration of a certain degree of immune-homeostasis and to a reduction of the severe imbalance produced by the exaggerated host response to bacterial invasion. Also, a personalized approach, matching RRT intensity with the risk of albumin and amino acid, catecholamine and antibiotic loss, should be advocated to avoid jeopardizing the beneficial effects of extracorporeal therapies [23]. The culture of this approach comes from the discipline of nephrology. For years, chronic haemodialysis has sustained thousands of lives even though a clear understanding of the molecular basis of uremia has not been achieved yet. A similar approach can be used for sepsis and multiple organ failure where altered blood composition represents the common ground for damage/dysfunction: whatever component is in excess or defect compared to its physiological concentration in blood, it can be removed or corrected by a specific extracorporeal treatment and device. This is the basis for the application of ECOS in critically ill patients (Fig. 2).

Kidney support can be provided by different intermittent and continuous blood purification techniques such as intermittent haemodialysis, slow low efficiency dialysis, continuous VV haemofiltration, haemodialysis, haemodiafiltration. These techniques, based on diffusive and/or convective transport of solutes and water transport by ultrafiltration across a semipermeable membrane, allow adequate blood purification, acid-base/electrolyte correction and volume control. In case of sepsis, such techniques are further expanded with the use of high-volume haemofiltration, coupled plasmafiltration adsorption and high-cut-off membrane applications. The last 2 techniques are also utilized when liver dysfunction or rhabdomyolysis are present and large molecular size molecules are to be removed from the circulation. In case of protein-bound solutes, albumin haemodialysis has also been suggested as well as plasmapheresis (PF) or plasma exchange (PE). A special nomenclature has been created to better define the characteristics of each component of the extracorporeal circuit [24] and each specific technique [25].

Adsorption has been proposed as a third mechanism for solute removal from the circulation. Sorbents prepared in specific cartridges can be placed in direct contact with blood as in the case of direct haemoperfusion (HP), or utilized after PF from whole blood (plasma filtration adsorption dialysis [PFAD]) to avoid direct contact of platelets and white cells with the sorbent particles. After plasma is processed in the sorbent bed, blood is reconstituted and returned to the patient. HP has been used for years in case of acute intoxication, hyperbilirubinemia and immunoadsorption. Recently, biocompatible sorbent devices have been created for endotoxin removal (polymixin B [PMX]-HP, Toray) or cytokine removal (cartridges for HP from Cytosorbents, Jaffron or others) in severe sepsis or septic shock.

Heart support has been originally achieved removing the excess of fluid in the body by ultrafiltration when diuretics cannot provide adequate diuresis. The spectrum of extracorporeal techniques has today expanded to other options. VA-ECMO is used in patients with acute cardiac or circulatory failure to restore end-organ perfusion and organ function, and to bridge either to recovery, to definite cardiac support (e.g., VADs) or heart transplantation. MODS is particularly common in patients requiring cardiac support and use of lung support and RRT may become additionally necessary [26]. Again VA-ECMO is part of ECOS because blood is processed outside the body while VAD, Total implantable heart, Impella or IABP technologies belong to the MOST category but not to ECOS.

Lung support in the context of ECOS has been traditionally identified with VV-ECMO. VV-ECMO is mostly used for correction of hypoxemia refractory to lung-protective ventilation and prone position in patients with severe acute respiratory distress syndrome. The experience coming again from haemodialysis brought into clinical practice, however, the possibility to achieve partial lung support with a certain removal of $\mathrm{CO}_{2}$ from the circulation. This concept of "respiratory dialysis" has further evolved to a system where a small oxygenator is placed in series with a continuous VV haemofiltration circuit [27]. The technique called $\mathrm{ECCO}_{2} \mathrm{R}$ is used as an alternative or supplement to MV for correction of hypercapnia, but not for blood oxygenation since the blood flows through the circuit are relatively low $(350-450 \mathrm{~mL} / \mathrm{min})$. Recently, RRT in conjunction with $\mathrm{ECCO}_{2} \mathrm{R}$ has been advocated to allow "super-protective" MV settings, and reduction of 
Fig. 2. Schematic representation of current ECOS techniques: ECCO2R, extracorporeal $\mathrm{CO}_{2}$ removal; VA-ECMO, venous arterial extracorporeal membrane oxygenation; SCUF, slow continuous ultrafiltration; CVVH, continuous veno-venous hemofiltration; CVVHD, continuous veno-venous hemodialysis; CVVHDF, continuous veno-venous Hemodiafiltration; SLED, sustained low efficiency hemodialysis; PF, plasmapheresis; PE, plasma exchange; HP, hemoperfusion; AHD, albumin hemodialysis; CPFA, continuous plasma filtration adsorption; VV-ECMO, veno venous extracorporeal membrane oxygenation. Minor modification of these seminal therapies have been reported in the literature but these represent the original concept.

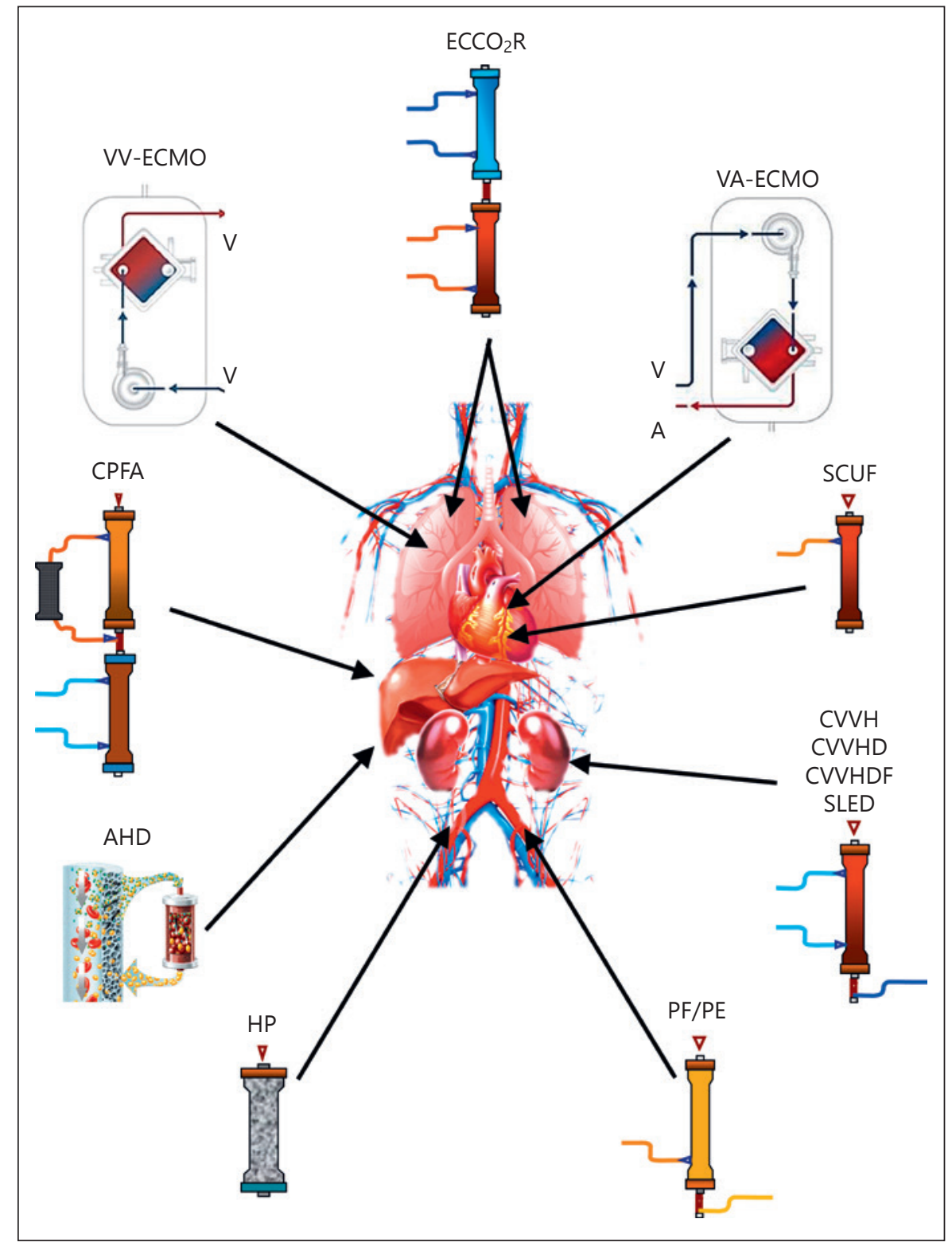

vasopressor demands in patients with ARDS experiencing AKI [28]. In some cases, $\mathrm{ECCO}_{2} \mathrm{R}$ can also allow the possibility to avoid invasive $\mathrm{MV}$ and continue with noninvasive mechanical ventilation (NIMV).

Liver support can be provided by albumin dialysis, plasmafiltration/adsorption, PE, and HP. Not only removal of bilirubin and other protein-bound toxins can be achieved by these techniques, but also significant reduction of ammonium level can be observed during treatment. MARS, Prometheus and other equipment based on cascade filtration and dialysis with albumin based dialysate and sorbents are currently available for this purpose [29].

From MOST to ECOS in Critically Ill Patients

\section{Native and Artificial Organ Cross-Talk}

There is a clear need to explore cross-talk and interactions between different organ systems in the critically ill patient. The literature on complex syndromes with multiorgan involvement emphasizes the need for multidisciplinary management. In these conditions, the level of multiple organ dysfunction makes MOST highly recommended or even mandatory. Frequently however, patients who display clear indication for ECMO and are undergoing such a complex therapy may require further organ support with the addition of RRT, liver support, HP for detoxification or cardiac support. In these circumstances, 
extracorporeal support and organ replacement may become safer and more uniform if different functions are combined in a fully integrated hardware. Fluid balance, solute removal, $\mathrm{CO}_{2}$ removal, aromatic amino acid removal, electrolyte and acid base equilibration, blood detoxification and oxygenation should be considered a continuum, where the artificial organ crosstalk is constant. Variations in $\mathrm{CO}_{2}$ must consider the use of buffers in dialysis or the application of citrate as anticoagulant for an adequate equilibrium of acid-base. The future is likely to see the introduction of a unified hardware with special circuitry that will facilitate the performance of all different organ support therapies on demand, simply escalating or de-escalating the complexity of the system. Thus, from ECMO and RRT, a patient may be progressively moved to $\mathrm{ECCO}_{2} \mathrm{R}$ and intermitted haemodialysis and, finally, may even be discharged with organ support including chronic haemodialysis and respiratory dialysis in case of non-recovery or progression towards chronic illness.

\section{The Next-Generation ECOS Equipment}

If MOST is applied and especially in the context of multipurpose ECOS, artificial organ crosstalk should be considered by a multidisciplinary task force to avoid negative interactions and unwanted side effects. An integrated monitoring of patients, chemistry and machine parameters will offer the basis for a "smart" biofeedback leading to correction in prescription and delivery of ECOS [30].

We strongly advocate the need for next-generation ECOS machines to achieve harmonization of components, techniques and operations of the multiple extracorporeal therapies. We suggest that simultaneous multiple functions and techniques be performed for optimizing artificial organ cross-talk while avoiding unwanted side effects or operational drawbacks due to poor integration of prescription and delivery parameters. Further studies are needed to establish the ideal timing of interventions, to find out whether early implementation impacts organ recovery and optimizes resource utilization, and to identify the patient groups that can be expected to benefit from long-term organ support.

\section{Acknowledgement}

This text and figures have been presented at the International Symposium on Intensive Care and Emergency Medicine held in Brussels in March 2018 and were reported in the magazine ICU Management for dissemination among congress participants.

\section{Disclosure Statement}

The authors do not have any conflicts of interest to disclose.

\section{References}

1 Kadri SS, Rhee C, Strich JR, Morales MK, Hohmann S, Menchaca J, Suffredini AF, Danner RL, Klompas M: Estimating ten-year trends in septic shock incidence and mortality in united states academic medical centers using clinical data. Chest 2017;151:278-285.

2 Kaukonen KM, Bailey M, Suzuki S, Pilcher D, Bellomo R: Mortality related to severe sepsis and septic shock among critically ill patients in Australia and New Zealand, 2000-2012. JAMA 2014;311:1308-1316.

3 Ziesmann MT, Marshall JC: Multiple organ dysfunction: the defining syndrome of sepsis. Surg Infect (Larchmt) 2018;19:184-190.

4 Kellum JA, Prowle JR: Paradigms of acute kidney injury in the intensive care setting. Nat Rev Nephrol 2018;14:217-230.

5 Husain-Syed F, McCullough PA, Birk HW, Renker M, Brocca A, Seeger W, Ronco C: Cardio-pulmonary-renal interactions: a multidisciplinary approach. J Am Coll Cardiol 2015;65:2433-2448.

6 Ronco C, Haapio M, House AA, Anavekar N, Bellomo R: Cardiorenal syndrome. J Am Coll Cardiol 2008;52:1527-1539.
7 McCullough PA, Kellum JA, Haase M, Müller C, Damman K, Murray PT, Cruz D, House AA, Schmidt-Ott KM, Vescovo G, Bagshaw SM, Hoste EA, Briguori C, Braam B, Chawla LS, Costanzo MR, Tumlin JA, Herzog CA, Mehta RL, Rabb H, Shaw AD, Singbartl K, Ronco C: Pathophysiology of the cardiorenal syndromes: executive summary from the eleventh consensus conference of the Acute Dialysis Quality Initiative (ADQI). Contrib Nephrol 2013;182:82-98.

8 Husain-Syed F, Slutsky AS, Ronco C: Lungkidney cross-talk in the critically Ill patient. Am J Respir Crit Care Med 2016;194:402414.

9 Siddiqui MS, Stravitz RT: Intensive care unit management of patients with liver failure. Clin Liver Dis 2014;18:957-978.

10 Fukazawa K, Lee HT: Updates on hepato-renal syndrome. J Anesth Clin Res 2013;4:352.

11 Arroyo V, Jiménez W: Complications of cirrhosis. II. Renal and circulatory dysfunction. Lights and shadows in an important clinical problem. J Hepatol 2000;32(1 suppl):157170.
12 Jacobs MC, Haak BW, Hugenholtz F, Wiersinga WJ: Gut microbiota and host defense in critical illness. Curr Opin Crit Care 2017;23: 257-263.

13 Doi K, Rabb H: Impact of acute kidney injury on distant organ function: recent findings and potential therapeutic targets. Kidney Int 2016; 89:555-564.

14 Rosenthal MD, Kamel AY, Rosenthal CM, Brakenridge S, Croft CA, Moore FA: Chronic critical illness: application of what we know. Nutr Clin Pract 2018;33:39-45.

15 Agarwal A, Dong Z, Harris R, Murray P, Parikh SM, Rosner MH, Kellum JA, Ronco C; Acute Dialysis Quality Initiative XIII Working Group: Cellular and molecular mechanisms of AKI. J Am Soc Nephrol 2016;27: 1288-1299.

16 Boomer JS, To K, Chang KC, Takasu O, Osborne DF, Walton AH, Bricker TL, Jarman SD 2nd, Kreisel D, Krupnick AS, Srivastava A, Swanson PE, Green JM, Hotchkiss RS: Immunosuppression in patients who die of sepsis and multiple organ failure. JAMA 2011;306: 2594-2605. 
17 Ronco C, Bellomo R: Acute renal failure and multiple organ dysfunction in the ICU: from renal replacement therapy (RRT) to multiple organ support therapy (MOST). Int J Artif Organs 2002;25:733-747.

18 Ronco C: Recent evolution of renal replacement therapy in the critically ill patient. Crit Care 2006;10:123.

19 Ranieri VM, Brodie D, Vincent JL: Extracorporeal organ support: from technological tool to clinical strategy supporting severe organ failure. JAMA 2017;318:1105-1106.

20 Tetta C, Cavaillon JM, Schulze M, Ronco C, Ghezzi PM, Camussi G, Serra AM, Curti F, Lonnemann G: Removal of cytokines and activated complement components in an experimental model of continuous plasma filtration coupled with sorbent adsorption. Nephrol Dial Transplant 1998;13:14581464.

21 Sieberth HG, Kierdorf HP: Is cytokine removal by continuous hemofiltration feasible? Kidney Int Suppl 1999;72:S79-S83.
22 Honore PM, Joannes-Boyau O, Merson L, Boer W, Piette V, Galloy AC, Janvier G: The big bang of hemofiltration: the beginning of a new era in the third millennium for extra-corporeal blood purification! Int J Artif Organs 2006;29:649-659.

23 Bagshaw SM, Chakravarthi MR, Ricci Z, Tolwani A, Neri M, De Rosa S, Kellum JA, Ronco C; ADQI Consensus Group: Precision continuous renal replacement therapy and solute control. Blood Purif 2016;42:238-247.

24 Neri M, Villa G, Garzotto F, et al: Nomenclature for renal replacement therapy and blood purification techniques in critically ill patients: basic principles. Crit Care 2016;20:318.

25 Villa G, Neri M, Bellomo R, et al: Nomenclature for renal replacement therapy and blood purification techniques in critically ill patients: practical considerations. Crit Care 2016;20:283.

26 Van Dorn CS, Aganga DO, Johnson JN: Extracorporeal membrane oxygenation, Berlin, and ventricular assist devices: a primer for the cardiologist. Curr Opin Cardiol 2018;33:8794.

27 Romagnoli S, Ricci Z, Ronco C: Novel extracorporeal therapies for combined renal-pulmonary dysfunction. Semin Nephrol 2016;36: 71-77.

28 Allardet-Servent J, Castanier M, Signouret T, Soundaravelou R, Lepidi A, Seghboyan JM: Safety and efficacy of combined extracorporeal $\mathrm{CO} 2$ removal and renal replacement therapy in patients with acute respiratory distress syndrome and acute kidney injury: the pulmonary and renal support in acute respiratory distress syndrome study. Crit Care Med 2015;43:2570-2581.

29 Faybik P, Krenn CG: Extracorporeal liver support. Curr Opin Crit Care 2013;19:149153.

30 Ricci Z, Romagnoli S, Ronco C: Automatic dialysis and continuous renal replacement therapy: keeping the primacy of human consciousness and fighting the dark side of technology. Blood Purif 2017;44:271-275. 